\title{
Juvenile Chronic Polyarthritis
}

National Cancer Institute

\section{Source}

National Cancer Institute. Juvenile Chronic Polyarthritis. NCI Thesaurus. Code C26979.

A group of conditions used to describe polyarthritis occurring in children. 